OPEN ACCESS

Edited by:

Bao-jun Sun,

Institute of Zoology, Chinese Academy of Sciences (CAS), China

Reviewed by:

Yang Wang,

Hebei Normal University, China

Shaobin Li,

Yangtze University, China

Guangzhan Fang,

Chinese Academy of Sciences (CAS),

China

*Correspondence:

Wen Bo Liao

Liaobo_0_0@126.com

${ }^{t}$ These authors have contributed

equally to this work

Specialty section:

This article was submitted to

Ecophysiology,

a section of the journal

Frontiers in Ecology and Evolution

Received: 09 August 2021

Accepted: 13 September 2021

Published: 01 October 2021

Citation:

Chen $C$, Jiang $Y$, Jin $L$ and

Liao WB (2021) No Evidence

for Effects of Ecological and Behavioral Factors on Eye Size

Evolution in Anurans.

Front. Ecol. Evol. 9:755818.

doi: 10.3389/fevo.2021.755818

\section{No Evidence for Effects of Ecological and Behavioral Factors on Eye Size Evolution in Anurans}

\author{
Chuan Chen ${ }^{1,2,3 \dagger}$, Ying Jiang ${ }^{1,2,3 \dagger}$, Long Jin ${ }^{1,2,3+}$ and Wen Bo Liao ${ }^{1,2,3 *}$ \\ ${ }^{1}$ Key Laboratory of Southwest China Wildlife Resources Conservation (Ministry of Education), China West Normal University, \\ Nanchong, China, ${ }^{2}$ Key Laboratory of Artificial Propagation and Utilization in Anurans of Nanchong City, China West Normal \\ University, Nanchong, China, ${ }^{3}$ Institute of Eco-adaptation in Amphibians and Reptiles, China West Normal University, \\ Nanchong, China
}

Eye size varies markedly among taxonomic levels, and this variation is often related to the patterns shaped by phylogeny and ecological and behavioral factors. The selective pressures underlying eye size evolution are especially studied in fishes, anurans, birds, and mammals. However, selective pressures underlying the eye size evolution in anurans have inconsistent scaling rules. Here, we investigated the links between eye size and both ecological (e.g., light availability and habitat type) and behavioral factors (e.g., activity time, foraging mobility, defensive strategy, and mating system) among 252 species of anurans by using phylogenetically controlled generalized leastsquared (PGLS) regression. Results show that anuran eye size scales hypo-allometrically with body size. However, eye size was not significantly influenced by ecological and behavioral factors, including habitat type, activity time, light availability, foraging mobility, defensive strategy, and mating system. Therefore, neither ecology nor behavior plays a key role in promoting eye size evolution in frogs.

Keywords: anurans, behavioral factors, body size, eye size, hypo-allometrical relationship

\section{INTRODUCTION}

The eyes can extract and exploit the information transmitted by light in animals at taxonomic groups (Land and Nilsson, 2012). Eye size varies extensively from tiny to giant among different animals (Martin, 1993; Land, 2009; Land and Nilsson, 2012). The size and dimension of an eye heavily affect the visual system and the abundance and quality of visual information, where animals extract from different environments (Walls, 1942). Although eye acuity is directly associated with eye size and lens diameter, it is also associated with other factors such as quality of the optic component, the angular spacing of the receptors, and the diameter of the photoreceptor (Veilleux and Kirk, 2014). Larger eyes possess larger abundance of photoreceptors and size of images, resulting in longer focal length for determining the retinal area size, where the images of the objects spread (Martin, 2007). Hence, selection for the increased resolving power leads to the enlarged eye size through the longer focal length (Martin, 1983).

While larger eyes can obtain information more effectively from changing environments compared with normal ones, eye size is physically and developmentally constrained (Huber et al., 1997; Moran et al., 2015). Considering the cost associated with benefiting from better vision by providing increased image resolution or enhance sensitivity to light, the eyes may reduce or even 
lose during over evolutionary periods in species that move into dark or murky ecosystems (Caves et al., 2017; Porter and SumnerRooney, 2018). In elasmobranchs, relative and absolute eye size varies considerably and species with smaller eyes tend to be costal, while species with larger eyes are oceanic species living in deep and dark environments (Lisney and Collin, 2007). Moreover, eye size is affected by the activity time, where nocturnal species have larger eye size associated with higher acuity than diurnal species (e.g., mammals: Veilleux and Kirk, 2014; birds: Garamszegi et al., 2002; Hall and Ross, 2007; geckos: Werner and Seifan, 2006).

Eye size is associated with behavioral factors, such as predator avoidance and prey tracking (Garamszegi et al., 2002; Møller and Erritzøe, 2010; Cronin et al., 2014; Thomas et al., 2020). For instance, predator pressure promotes eye size evolution in preys, because the better processing of visual information in species with large eyes enhance the survivability of birds and fishes (Douglas and Hawryshyn, 1990; Land and Nilsson, 2012; Starunov et al., 2017). Moreover, the cutaneous secretion of toxin is one of the main defensive mechanisms against predator risk, and species with granular glands may experience lower predator risk in anurans (Toledo and Jared, 1995; Prates et al., 2012; Huang et al., 2019). As a result, species with granular glands are associated with smaller eye size compared with species with non-granular glands.

For anurans, investigating the relationships of eye size and both ecological and behavioral factors among 44 species have revealed that eye size is not associated with activity time, foraging mobility, habitat type, defensive strategy, mating system, and water turbidity (Huang et al., 2019). However, a recent study has evaluated the eye size and six traits of natural history hypothesized to be related to eye size evolution among 220 species of anurans, and the results indicate that eye size is correlated with adult mating habitat and activity patterns (Thomas et al., 2020). Although anuran eye size was studied outside of a few families, potential relationships between eye size and ecological and behavioral factors are unclear.

In the present study, we investigated the relationships between eye size and both ecological (e.g., light availability and habitat type) and behavioral factors (e.g., defensive strategy, activity time, foraging mobility, and mating system) in 252 anuran species within seven families in China. First, we predicted that species living in aquatic habitats would display increased eye size, because they inhabit light environments. Second, we predicted that nocturnal species would have large eyes to maximize sensitivity in low-light conditions. Third, we predicted that species approaching slowly and capturing prey would have smaller eyes than species approaching quickly and often chasing prey. Finally, we predicted that species with granular glands would possess small eyes, because they experience low predator risk.

\section{MATERIALS AND METHODS}

\section{Data Collection}

The data of eye diameter and snout-vent length (SVL) were collected from "Fauna Sinica," which includes 3,171 males and
1,820 females in 252 anuran species (Fei et al., 2009). We calculated the average eye size and SVL based on males and females for all analyses (Supplementary Table 1). All species in this study have available phylogenetic information.

\section{Categorical Variables}

Following the method of Thomas et al. (2020) and our filed observation, adult habitat type for each species on a six-point scale were classified into scansorial [primarily associated with plants; up off the ground (arboreal/shrubs/reeds)], ground-dwelling (primarily active on the ground), subfossorial (primarily active under leaf litter; shallow burrowers), fossorial [primarily active in deeper burrows (not burrowing simply for aestivation/long periods of inactivity)], aquatic (primarily found in water; rarely/never leaves water), and semi-aquatic (strongly associated with/commonly found in water but also frequently uses land habitats). Activity time was classified on a two-point scale, namely, nocturnal (primarily active at night), and both diurnal and nocturnal (active during both day and at night) (Huang et al., 2019). Foraging mobility was categorized based on behavioral phenotype, including species approaching slowly and capturing prey and species approaching quickly and often actively chasing prey (Huang et al., 2019). Light availability of species was classified into strong light (i.e., forest is an open environment) and weak light (i.e., light does not penetrate much) (Huang et al., 2019). Defensive strategy was also classified on a two-point scale, namely, species with granular glands in dorsal and ventral skin and species with non-granular glands in skin (Huang et al., 2019). Mating system for each species was classified as polyandry and monandry (Zeng et al., 2016).

\section{Phylogeny}

The molecular phylogeny was constructed in the 252 species based on three mitochondrial genes and three nuclear genes (Figure 1). The mitochondrial genes included the large and small subunits of the mitochondrial ribosome genes (12S/16S) and cytochrome b (CYTB). The nuclear genes included the rhodopsin (RHOD), the tyrosinase (TYR), and the recombination-activating gene 1 (RAG1). All sequences were aligned using the MUSCLE function in MEGA v.6.0.6 (Tamura et al., 2013), and we determined the best nucleotide substitution model of each gene by using the Akaike Information Criterion in jModelTest v.2.1.2 (Darriba et al., 2012). The best substitution model was TYR, HKY + G for RAG1 and RHOD, GTR + G for $12 \mathrm{~S}$ and GTR + I + G for CYTB and 16S. Considering the lack of fossil dates, we used these models to construct the phylogenies based on BEAUTi and BEAST v.1.8.3 (Drummond et al., 2012; Mai et al., 2019; Mai et al., 2020), with a relaxed uncorrelated lognormal clock, unlinked substitution models, a Yule speciation process, and no calibration points. The effective sample size values for each tree statistics showed the satisfying convergence of the Bayesian chain and adequate model mixing in Tracer v.1.6.0 (Rambaut and Drummond, 2014). Then, we generated a maximum clade credibility tree with a $20 \%$ burn-in and mean node heights before ending the analysis by using TreeAnnotator v.1.8.3 (Drummond et al., 2012; Chen et al., 2021). 


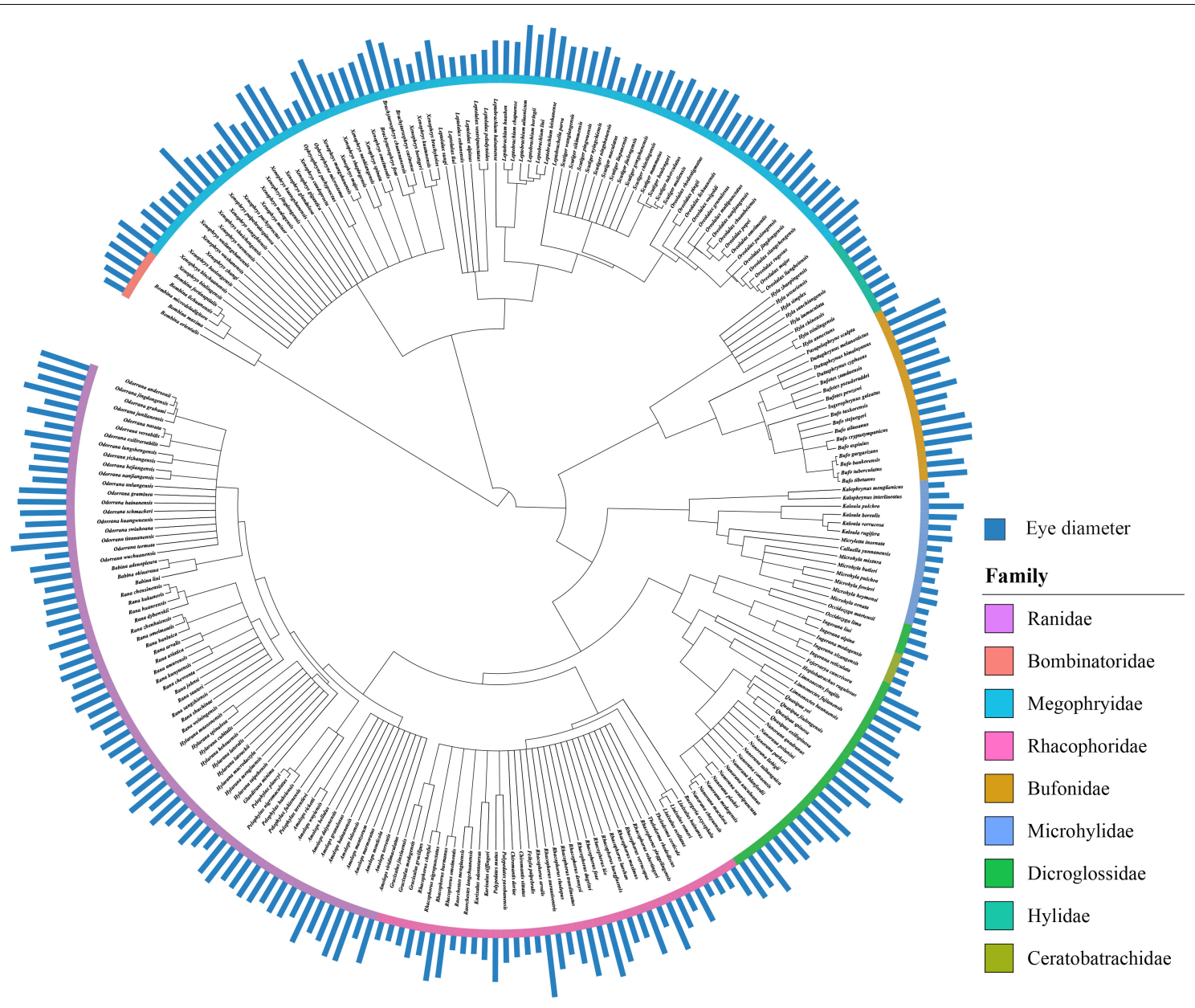

FIGURE 1 | Phylogenetic tree of the 252 Chinese anuran species on the basis of the three nuclear genes (RAG1, RHOD, TYR) and the three mitochondrial genes (CYTB, 12S, 16S) using TreeAnnotator v.1.8.3 in the comparative analysis. Phylogeny shows means of eye diameter in anurans of species. The color indicates family in frogs.

\section{Data Analysis}

All data analysis were performed in the statistical software $\mathrm{R}$ v. 4.1.0 (R Development Core Team, 2021). We analyzed the evolutionary link between eye and body size among species by using phylogenetically controlled generalized least-squared (PGLS) regression with $\log _{10}$-transformed data in the APEpackage in $\mathrm{R}$ software (v. 5.4-1) (Paradis, 2012; Cai et al., 2020; Huang et al., 2020). In PGLS analysis, we estimated the phylogenetic scaling parameter $(\lambda)$ by using the maximumlikelihood method. In the model residuals, $\lambda$ represented the phylogenetic signal. The parameter $\lambda$ estimated the effect of phylogenetic signal on the relationship between eye and body size $(\lambda=1$ indicates strongly phylogenetic signal, and $\lambda=0$ indicates no phylogenetic signal). For comparison with allometric studies, where the phylogenetical relationships were corrected, we used the ordinary least-squares (OLS) regressions in stats v. 4.1.0 ( $\mathrm{R}$ Development Core Team, 2021) and the standardized major axis (SMA) regressions in smatr v. 3.4.8 (Warton et al., 2012) with the same $\log _{10}$-transformed data.
For testing the effect of ecological and behavioral factors on eye size, we used multiparameter fixed analysis in PGLS regression to test whether variables were associated with eye size. To test the effect of each of ecological and behavioral factors on eye size evolution, we first use phylogenetic ANCOVAs conducted through caper package of PGLS models with SVL as a covariate (e.g., ED SVL* habitat type) and adult habitat, activity time, foraging mobility, light availability, defensive strategy or mating system as a fixed effect (Thomas et al., 2020). Besides, we ran three models (including OU, BM, and Pagel) in PGLS for each variable to avoid overparameterizing model data redundancy (Thomas et al., 2020).

\section{RESULTS}

\section{Eye Size Scales Hypo-Allometrically With Snout-Vent Length}

A hypo-allometric (slope $<1$ ) interspecific scaling was observed between eye size and SVL in 252 species of anurans (PGLS: 
slope $=0.818, R^{2}=0.821, P<0.0001, \lambda=0.415$; Figure 2). The OLS and SMA models produced similar fits to PGLS models, though the slopes of SMA models were the highest (slope $=0.889$, $P<0.001)$, whereas those of PGLS were the lowest. SMA tests also indicated that the scaling of eye size with SVL was hypo-allometric.

\section{Eye Size Correlates With Ecological and Behavioral Traits}

Analysis with phylogenetic ANCOVA in separate models showed that the relative eye size was not affected by habitat type (Supplementary Table $2 ; F_{1}<0.001, p=0.988$ ), activity time
$\left(F_{1}=0.128, p=0.721\right)$, foraging mobility $\left(F_{1}=0.017, p=0.897\right)$, light availability $\left(F_{1}=0.996, p=0.319\right)$, defensive strategies $\left(F_{1}=0.632, p=0.427\right)$, and mating system $\left(F_{1}<0.001,0.992\right)$.

PGLS in a single model revealed that the relative eye size was not affected significantly by habitat type, activity time, light availability, foraging mobility, defensive strategy, and mating system (Table 1). Nocturnal species did not possess larger eyes than diurnal and nocturnal species (Figure 3A). Species approaching slowly and capturing prey did not have smaller eyes than species approaching quickly and often actively chasing prey (Figure 3B). Species living under strong light did not have smaller eyes than species living in weak light (Figure 3C). Species with granular glands in dorsal and ventral skin did not have smaller

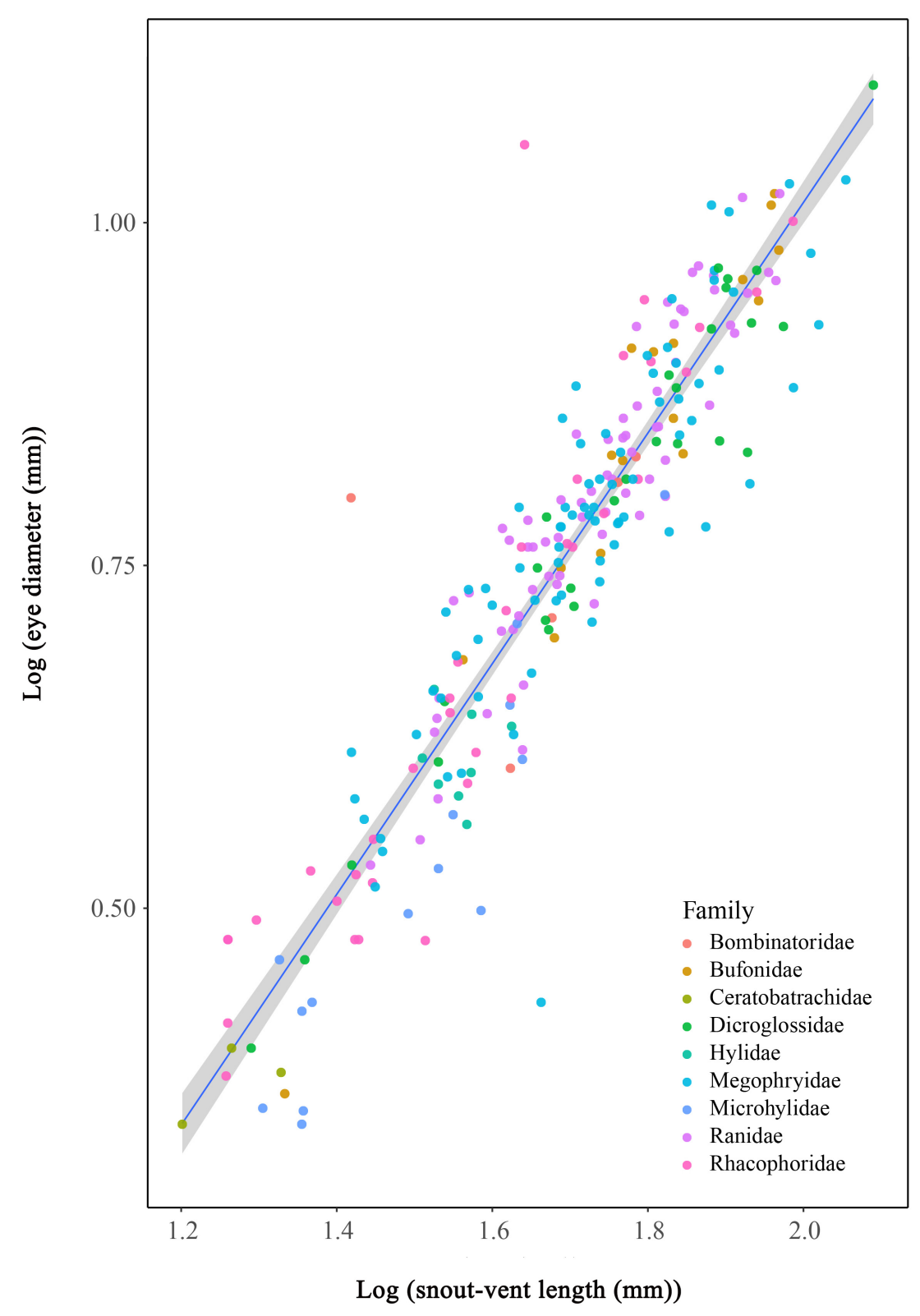

FIGURE 2 | Hypo-allometrical relationships between eye diameter and snout-vent length across 252 anurans species. 
TABLE 1 | Evolution of eye size in relation to various predictor variables across 252 anurans species using phylogenetic generalized least squares model.

\begin{tabular}{lrrrrr}
\hline Predictors & \multicolumn{5}{c}{ Eye size } \\
& $\boldsymbol{\lambda}$ & $\boldsymbol{\beta}$ & $\boldsymbol{t}$ & $\boldsymbol{R}^{\mathbf{2}}$ & $\boldsymbol{P}$ \\
\cline { 2 - 6 } & $0.3800^{1,<0.001}$ & -0.0004 & -0.1461 & 0.0001 & 0.8840 \\
\hline Habitat type & -0.0018 & -0.2161 & 0.0002 & 0.8291 \\
Activity time & 0.0078 & 0.7680 & 0.0024 & 0.4432 \\
Light availability & & 0.0045 & 0.3501 & 0.0005 & 0.7266 \\
Foraging mobility & -0.0086 & -0.7629 & 0.0024 & 0.4463 \\
Defensive strategy & & 0.0019 & 0.0753 & 0.00002 & 0.9400 \\
Mating system & & 0.8157 & 31.4329 & 0.8019 & $<0.001$ \\
SVL & & & & & \\
\hline
\end{tabular}

eyes than species with non-granular glands in skin (Figure 3D). The effects of habitat type and mating system on eye size is shown in Supplementary Figures 1A,B.

\section{DISCUSSION}

Eye size scales with body size among 252 species of anurans in this study, in which species with larger bodies have larger eye size, indicating a hypo-allometric relationship between body and eye size. However, eye size in anurans is not affected by ecological and behavioral factors (e.g., adult mating habitats, activity time, foraging mobility, light availability, defensive strategy, and mating system). Scansorial species do not have larger eyes than ground-dwelling, fossorial, and aquatic species. Nocturnal species living under weak light do not possess larger eyes than both nocturnal and diurnal species living under strong light. Moreover, species approaching quickly and often actively chasing preys do not display larger eyes than species approaching slowly preys. Moreover, species with granular glands did not have larger eyes than those without granular glands. Hence, these traits are not correlated with eye size when correcting for body size and phylogeny, suggesting that light availability and foraging behavior cannot play a key role in shaping eye size evolution in anurans.

Body size is the strongest predictor of eye size, which is expected as larger species usually possess larger eyes than smaller species in sharks, reptile, birds, and mammals (Brooke et al., 1999; Kiltie, 2000; Garamszegi et al., 2002; Ross et al., 2006; Werner and Seifan, 2006; Lisney and Collin, 2007; Liu et al., 2012). For anurans, eye size scales hypo-allometrically with SVL (Huang et al., 2019; Thomas et al., 2020) and we found the same patterns as Huang' and Thomas' that larger species had larger eyes, displaying hypo-allometrical relationship between eye size and body size.

Eye size varied among adult habitats in animal groups. For instance, evidence supports that larger eye in anurans are beneficial to scansorial species by accommodating fast temporal resolutions during jumping process and high acuity in visually complex arboreal habitats (Thomas et al., 2020). Likewise, fossorial species reduced eye size as adaptations to dark and/or abrasive habitats in mammals (Borghi et al., 2002), fishes (Eagderi and Adriaens, 2010), caecilian amphibians (Mohun et al., 2010), and reptiles (Yovanovich et al., 2019).
However, the variation in the eye size of the 44 species of anurans investigated seems largely independent of adult habitats, possibly because all specimens were obtained from one, albeit large, geographical region (Huang et al., 2019). In the present study, eye size did not increase from fully fossorial to subfossorial to non-fossorial (e.g., grounddwelling, semiaquatic, scansorial) species, indicating that the fossoriality degree cannot determine eye size evolution across 252 species of anurans.

Many nocturnal vertebrates display enlarged eye size to maximize visual sensitivity (e.g., primates: Kirk, 2006; birds: Hall and Ross, 2007; reef fish: Schmitz and Wainwright, 2011). For instance, nocturnal birds evolve larger eyes to provide a wide pupil and improve light sensitivity during night compared with diurnal birds (Martin, 1985; Brooke et al., 1999). Significant influences were observed in the activity pattern of relative eye size among the 220 species of anurans, in which nocturnal species evolved larger eyes compared with both diurnal species and nocturnal and diurnal species (Thomas et al., 2020). However, previous studies have indicated that activity pattern cannot affect variation in relative eye size among species because most of the 44 species of anurans can be seen foraging and mating also at night (Huang et al., 2019; Mai et al., 2020). Similar to the findings of Huang et al. (2019), we found that activity pattern did not affect relative eye size among the 252 species of anurans, possibly because the common anuran ancestor is likely to be nocturnal, and the majority of the species of extant anurans retain this active pattern (Anderson and Wiens, 2017). Large eyes are associated with larger abundance of photoreceptors and produce larger image sizes, thereby collecting more light per solid angle of image than small eyes (Martin, 2007). For fishes, the larger eyes increase the chance of photon capture when detecting small bioluminescent flashes at low levels of sunlight (Warrant, 2000; Warrant and Locket, 2004). Moreover, to improve light sensitivity at night, it may be beneficial to enlarge not only retinal area, but also pupil diameter in dim light conditions (Martin, 1985). However, light availability did not affect the eye size evolution in anurans, where species living under weak light did not have larger eyes than those living under strong light. Hence, nocturnal species with higher light availability did not evolve larger eyes than diurnal species, suggesting that light availability was not correlated with detection of predator risks.

Vision plays an important role in searching for foods for birds, as indicated by marked differences in eye size across the types of foraging mobility, where species approaching quickly and actively capturing preys displayed an increase in eye size, whereas those approaching slowly preys displayed a decrease in eye size (Garamszegi et al., 2002). In a previous study on 44 species of anurans, foraging mobility did not affect eye size evolution, suggesting that anurans approaching quickly preys may live in low-light conditions, which cannot lead to increased eye size compared with anurans approaching quickly preys (Huang et al., 2019). All the 44 used species are possibly at least partially active at night. However, after sampling a larger number of species in our study, the results did not confirm the positive effect of capture behavior on eye size evolution. 

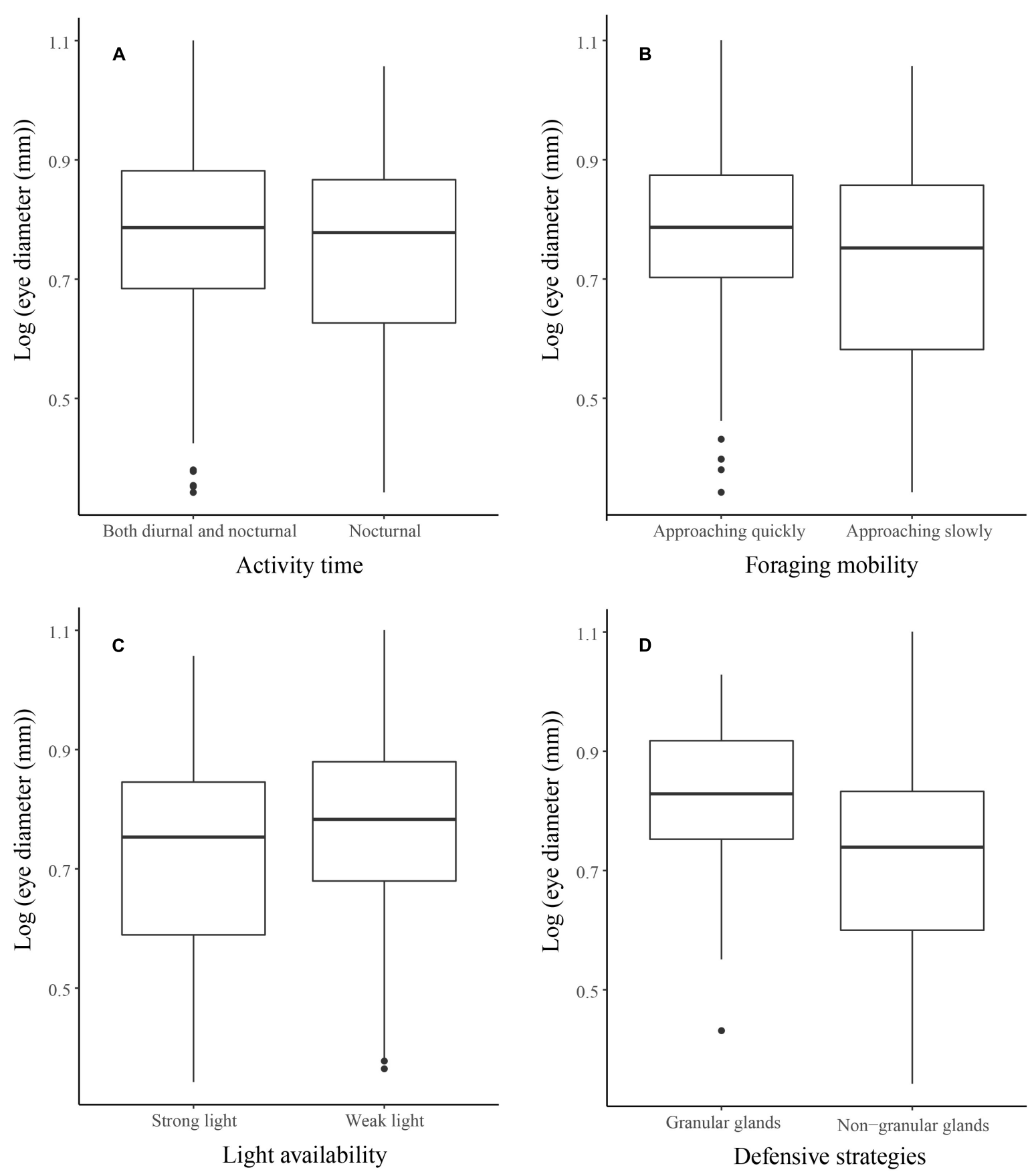

FIGURE 3 | Relationships between eye diameter and (A) activity time, (B) foraging mobility, (C) light availability, and (D) defensive strategies across 252 anuran species. Black points indicate the outliers.

Predator risk is the strongest selective pressure in shaping eye size evolution (Garamszegi et al., 2002). Considering species with larger eyes can easily spot predators early (Striedter, 2005; Kotrschal et al., 2015), stronger predator risk coincides with the larger-eyed birds (Møller and Erritzøe, 2010). The relationship between eye size and predator risk may be as prominent as it is for instance in birds with eye size that is positively correlated with fight distance (Blumstein et al., 2004). Consistent with a previous study (Huang et al., 2019), we found that species with granular glands in skin did not exhibit smaller eyes than species with non-granular glands in skin, suggesting that species depending on chemical defense cannot shape eye size evolution in anurans. Moreover, relative eye size is neither correlated with sexual size dimorphism nor mating system among the 44 species of anurans (Huang et al., 2019). In the present study, we found that eye size variation in 252 species of anuran cannot be explained by 
mating system contrary to the prominent role of sexual selection in eye size evolution.

In conclusion, eye size variation cannot be explained by ecological and behavioral factors including activity time, light availability, foraging mobility, and defensive strategy in our sample of 252 Chinese frogs. Eye size is not correlated with activity pattern and light availability, showing that a potential nocturnal bottleneck is not observed in anuran eyes evolution. Moreover, foraging mobility is not associated with the eye size in anurans, highlighting that foraging behavior cannot shape eye size evolution. Considering that defensive strategy (i.e., poison glands) does not affect eye size, the anti-predator ability in anurans cannot promote the enlarged eye size.

\section{DATA AVAILABILITY STATEMENT}

The original contributions presented in the study are included in the article/Supplementary Material, further inquiries can be directed to the corresponding author/s.

\section{ETHICS STATEMENT}

The animal study was reviewed and approved by China West Normal University.

\section{REFERENCES}

Anderson, S. R., and Wiens, J. J. (2017). Out of the dark: 350 million years of conservatism and evolution in diel activity patterns in vertebrates. Evolution 71, 1944-1959. doi: 10.1111/evo.13284

Blumstein, D. T., Fernández-Juricic, E., LeDee, O., Larsen, E., Rodriguez-Prieto, I. and Zugmeyer, C. (2004). Avian risk assessment: effects of perching height and detectability. Ethology 110, 273-285. doi: 10.1111/j.1439-0310.2004.00970.x

Borghi, C. E., Giannoni, S. M., and Roig, V. G. (2002). Eye reduction in subterranean mammals and eye protective behavior in Ctenomys. Mastozool. Neotrop. 9, 123-134.

Brooke, M. D., Hanley, S., and Laughlin, S. B. (1999). The scaling of eye size with body mass in birds. Proc. R. Soc. B 266, 405-412. doi: 10.1098/rspb.1999.0652

Cai, L., Mai, C. L., Yu, J. P., and Liao, W. B. (2020). The effects of prey items diversity and digestible materials in stomach on digestive tract length in Hylarana guentheri. Asian Herpetol. Res. 11, 155-160. doi: 10.16373/j.cnki.ahr. 190043

Caves, E., Sutton, T. T., and Johnsen, S. (2017). Visual acuity in ray-finned fishes correlates with eye size and habitat. J. Exp. Biol. 220, 1586-1596. doi: 10.1242/ jeb. 151183

Chen, C., Jin, L., Jiang, Y., and Liao, W. B. (2021). Effects of life histories on genome size variation in Squamata. Asian Herpetol. Res. doi: 10.16373/j.cnki.ahr.210018

Cronin, T. W., Johnsen, S., Marshall, N. J., and Warrant, E. J. (2014). Visual Ecology. Princeton, NJ: Princeton University Press. doi: 10.1515/9781400853021

Darriba, D., Taboada, G. L., Doallo, R., and Posada, D. (2012). jModelTest 2: more models, new heuristics and parallel computing. Nat. Methods 9:772. doi: 10.1038/nmeth.2109

Douglas, R. H., and Hawryshyn, C. W. (1990). "Behavioural studies of fish vision: an analysis of visual capabilities," in The Visual System of Fish, eds R. H. Douglas, and M. B. A. Djamoz (Dordrecht: Springer), 373-418. doi: 10.1007/978-94009-0411-8_11

Drummond, A. J., Suchard, M. A., Xie, D., and Rambaut, A. (2012). Bayesian phylogenetics with BEAUti and the BEAST 1.7. Mol. Biol. Evol. 29, 1969-1973. doi: $10.1093 / \mathrm{molbev} / \mathrm{mss} 075$

\section{AUTHOR CONTRIBUTIONS}

YJ and CC participated in laboratory work, data analysis, and manuscript drafting. LJ and WBL conducted data analysis and visual representation of the data. All authors contributed to the article and approved the submitted version.

\section{FUNDING}

Financial support was provided by the National Natural Sciences Foundation of China (31772451 and 31970393) and the Science and Technology Youth Innovation Team of Sichuan Province (2019JDTD0012).

\section{ACKNOWLEDGMENTS}

We would like to thank Li Zhao, Zhiping Mi, and Shengnan Chen for their assistance in data collection during the experiment.

\section{SUPPLEMENTARY MATERIAL}

The Supplementary Material for this article can be found online at: https://www.frontiersin.org/articles/10.3389/fevo.2021. 755818/full\#supplementary-material

Eagderi, S., and Adriaens, D. (2010). Cephalic morphology of Pythonichthys macrurus (Heterenchelyidae: Anguilliformes): specializations for headfirst burrowing. J. Morphol. 271, 1053-1065. doi: 10.1002/jmor. 10852

Fei, L., Hu, S. Q., Ye, C. Y., Huang, Y. Z., et al. (2009). Fauna Sinica (Amphibia Volume 2-3, Anura). Beijing: Science Press.

Garamszegi, L. Z., Møller, A. P., and Erritzøe, J. (2002). Coevolving avian eye size and brain size in relation to prey capture and nocturnality. Proc. R. Soc. B. 269, 961-967. doi: 10.1098/rspb.2002.1967

Hall, M. I., and Ross, C. F. (2007). Eye shape and activity pattern in birds. J. Zool. 271, 437-444. doi: 10.1111/j.1469-7998.2006.00227.x

Huang, C. H., Zhong, M. J., Liao, W. B., and Kotrschal, A. (2019). Investigating the role of body size, ecology, and behavior in anuran eye size evolution. Evol. Ecol. 33, 585-598. doi: 10.1007/s10682-019-09993-0

Huang, Y., Mai, C. L., Liao, W. B., and Kotrschal, A. (2020). Body mass variation is negatively associated with brain size-evidence for the fat-brain trade-off in anurans. Evolution 74, 1551-1557. doi: 10.1111/evo.13991

Huber, R., van Staaden, M., Kaufman, L. S., and Liem, K. F. (1997). Microhabitat use, trophic patterns and the evolution of brain structure in African cichlids. Brain Behav. Evol. 50, 167-182. doi: 10.1159/000113330

Kiltie, R. A. (2000). Scaling of visual acuity with body size in mammals and birds. Funct. Ecol. 14, 226-234. doi: 10.1046/j.1365-2435.2000. 00404.x

Kirk, E. C. (2006). Effects of activity pattern on eye size and orbital aperture size in primates. J. Hum. Evol. 51, 159-170. doi: 10.1016/j.jhevol.2006.02.004

Kotrschal, A., Buechel, S. D., Zala, S. M., Corral-Lopez, A., Penn, D. J., and Kolm, N. (2015). Brain size affects female but not male survival under predation threat. Ecol. Lett. 18, 646-652. doi: 10.1111/ele.12441

Land, M. F. (2009). Vision, eye movements, and natural behavior. Vis. Neurosci. 26, 51-62. doi: 10.1017/s0952523808080899

Land, M. F., and Nilsson, D. E. (2012). Animal Eyes. Oxford: Oxford University Press.

Lisney, T. J., and Collin, S. P. (2007). Relative eye size in elasmobranchs. Brain Behav. Evol. 69, 266-279. doi: 10.1159/000100036 
Liu, Y., Ding, L., Lei, J., Zhao, E. M., and Tang, Y. Z. (2012). Eye size variation reflects habitat and daily activity patterns in colubrid snakes. J. Morphol. 273, 883-893. doi: 10.1002/jmor.20028

Mai, C. L., Liao, W. B., Lüpold, S., and Kotrschal, A. (2020). Relative brain size is predicted by the intensity of intrasexual competition in frogs. Am. Nat. 196, 169-179. doi: 10.1086/709465

Mai, C. L., Yu, J. P., and Liao, W. B. (2019). Ecological and geographical reasons for the variation of digestive tract length in anurans. Asian Herpetol. Res. 10, 246-252. doi: 10.16373/j.cnki.ahr.190037

Martin, G. R. (1983). "Schematic eye models in vertebrates," in Progress in Sensory Physiology, ed. A. Gallego (Berlin: Springer), 43-81. doi: 10.1007/978-3-64269163-8_2

Martin, G. R. (1985). "Eye," in Form and Function in Birds, Vol. 3, eds A. S. King, and J. McClelland (London: Academic Press), 311-373.

Martin, G. R. (1993). "Producing the image," in Vision Brain, and Behavior in Birds, eds H. P. Zeigler, and H. J. Bischof (Cambridge, MA: MIT Press), 5-24.

Martin, G. R. (2007). Visual fields and their functions in birds. J. Ornthol. 148, 547-562. doi: 10.1007/s10336-007-0213-6

Mohun, S. M., Davies, W. L., Bowmaker, J. K., Pisani, D., Himstedt, W., Gower, D. J., et al. (2010). Identification and characterization of visual pigments in caecilians (Amphibia: Gymnophiona), an order of limbless vertebrates with rudimentary eyes. J. Exp. Biol. 213, 3586-3592. doi: 10.1242/jeb.045914

Møller, A. P., and Erritzøe, J. (2010). Flight distance and eye size in birds. Ethology 116, 458-465. doi: 10.1111/j.1439-0310.2010.01754.x

Moran, D., Softley, R., and Warrant, E. J. (2015). The energetic cost of vision and the evolution of eyeless Mexican cavefish. Sci. Adv. 1:e1500363. doi: 10.1126/ sciadv. 1500363

Paradis, E. (2012). Analysis of Phylogenetics and Evolution with R, Vol. 2. New York, NY: Springer.

Porter, M. L., and Sumner-Rooney, L. (2018). Evolution in the dark: unifying our understanding of eye loss. Integr. Comp. Biol. 58, 367-371. doi: 10.1093/icb/ icy082

Prates, I., Antoniazzi, M. M., Sciani, J. M., Pimenta, D. C., Toledo, L. F., Haddad, C. F., et al. (2012). Skin glands, poison and mimicry in dendrobatid and leptodactylid amphibians. J. Morphol. 273, 279-290. doi: 10.1002/jmor. 11021

R Development Core Team (2021). R: A Language and Environment for Statistical Computing. Vienna: R Development Core Team.

Rambaut, A., and Drummond, A. (2014). Tracer v1.6. Available online at: https: //tree.bio.ed.ac.uk/software/tracer/ (accessed August 4, 2021).

Ross, C. F., Hall, M. I., and Heesy, C. P. (2006). "Were basal primates nocturnal? Evidence from eye and orbit shape," in Primate Origins and Adaptations, eds M. Ravosa, and M. Dagosto (New York, NY: Kluwer), 233-256. doi: 10.1007/9780-387-33507-0_7

Schmitz, L., and Wainwright, P. C. (2011). Nocturnality constrains morphological and functional diversity in the eyes of reef fishes. BMC Evol. Biol. 11:338. doi: 10.1186/1471-2148-11-338

Starunov, V. V., Voronezhskaya, E. E., and Nezlin, L. P. (2017). Development of the nervous system in Platynereis dumerilii (Nereididae, Annelida). Front. Zool. 14:27. doi: 10.1186/s12983-017-0211-3
Striedter, G. F. (2005). Principles of Brain Evolution. Sunderland, MA: Sinauer associates Inc.

Tamura, K., Stecher, G., Peterson, D., Filipski, A., and Kumar, S. (2013). MEGA6: molecular evolutionary genetics analysis version 6.0. Mol. Biol. Evol. 30, 27252729. doi: 10.1093/molbev/mst197

Thomas, K. N., Gower, D. J., Bell, R. C., Fujita, M. K., and Streicher, J. W. (2020). Eye size and investment in frogs and toads correlate with adult habitat, activity pattern and breeding ecology. Proc. R. Soc. B 287:20201393. doi: 10.1098/rspb. 2020.1393

Toledo, R. D., and Jared, C. (1995). Cutaneous granular glands and amphibian venoms. Comp. Biochem. Physiol. A 111, 1-29. doi: 10.1016/0300-9629(95) 98515-i

Veilleux, C. C., and Kirk, E. C. (2014). Visual acuity in mammals: effect of eye size and ecology. Brain Behav. Evol. 83, 43-53. doi: 10.1159/000357830

Walls, G. L. (1942). The Vertebrate Eye and its Adaptive Radiation. New York, NY: Hafner.

Warrant, E. (2000). The eyes of deep-sea fishes and the changing nature of visual scenes with depth. Philos. Trans. R. Soc. B 355, 1155-1159. doi: 10.1098/rstb. 2000.0658

Warrant, E. J., and Locket, N. A. (2004). Vision in the deep sea. Biol. Rev. 79, $671-712$.

Warton, D. I., Duursma, R. A., Falster, D. S., and Taskinen, S. (2012). smatr 3-an R package for estimation and inference about allometric lines. Methods Ecol. Evol. 3, 257-259. doi: 10.1111/j.2041-210x.2011.00153.x

Werner, Y. L., and Seifan, T. (2006). Eye size in geckos: asymmetry, allometry, sexual dimorphism, and behavioral correlates. J. Morphol. 267, 1486-1500. doi: 10.1002/jmor.10499

Yovanovich, C. A., Pierotti, M. E., Rodrigues, M. T., and Grant, T. (2019). A dune with a view: the eyes of a neotropical fossorial lizard. Front. Zool. 16:17. doi: 10.1186/s12983-019-0320-2

Zeng, Y., Lou, S. L., Liao, W. B., Jehle, R., and Kotrschal, A. (2016). Sexual selection impacts brain anatomy in frogs and toads. Ecol. Evol. 6, 7070-7079. doi: $10.1002 /$ ece 3.2459

Conflict of Interest: The authors declare that the research was conducted in the absence of any commercial or financial relationships that could be construed as a potential conflict of interest.

Publisher's Note: All claims expressed in this article are solely those of the authors and do not necessarily represent those of their affiliated organizations, or those of the publisher, the editors and the reviewers. Any product that may be evaluated in this article, or claim that may be made by its manufacturer, is not guaranteed or endorsed by the publisher.

Copyright (c) 2021 Chen, Jiang, Jin and Liao. This is an open-access article distributed under the terms of the Creative Commons Attribution License (CC BY). The use, distribution or reproduction in other forums is permitted, provided the original author(s) and the copyright owner(s) are credited and that the original publication in this journal is cited, in accordance with accepted academic practice. No use, distribution or reproduction is permitted which does not comply with these terms. 